\title{
Effect of external electric field on Cyclodextrin-Alcohol adducts: A DFT study
}

\author{
KUNDAN BARUAH and PRADIP KR BHATTACHARYYA* \\ Department of Chemistry, Arya Vidyapeeth College, Guwahati 781 016, Assam, India \\ e-mail: prdpbhatta@yahoo.com
}

MS received 16 November 2014; revised 20 February 2015; accepted 28 February 2015

\begin{abstract}
Effect of external electric fields on the interaction energy between cyclodextrin and alcohol was analyzed in the light of density functional theory (DFT) and density functional reactivity theory (DFRT). Stability of the cyclodextrin-alcohol adducts was measured in terms of DFT based reactivity descriptor, global hardness, electrophilicity, and energy of the HOMO. Stability of adducts was observed to be sensitive towards the strength as well as direction of the applied external electric field. In addition, reactivity pattern follows the maximum hardness and minimum electrophilicity principles.
\end{abstract}

Keywords. Membrane; electric field; DFT; reactivity descriptor; MHP.

\section{Introduction}

Cyclodextrins (CDs) are natural biomolecules which are derived from starch and have a remarkable capacity to form inclusion complexes in solution or in the solid state with organic molecules through host-guest interactions with unique self assembling behaviour. ${ }^{1}$ The glucose units in CDs are arranged in such a way that hydroxymethylene groups are pointing downwards, whereas hydroxyl groups are pointing upwards. As a result of this, a hydrophilic outer space and a hydrophobic inner space are formed leading to the particular ability of the CDs to form host-guest complexes with organic components having appropriate diameters and physical interactions.

Combination of host-guest complex formation ability and structural features of CDs make them as novel polymer for membrane preparations, which are used extensively in separation science offering a good choice for separation of compounds and extraction processes. ${ }^{2}$ CDs are very attractive components for the enhancement of the selectivity for a specific membrane polymer towards aromatic/aliphatic components. ${ }^{3}$ During filtration process, interaction between the membrane components and substrates is important. Usually, stronger adsorption of the substrate slows down the filtration process. Strong interactions (hydrogen bonding or electrostatic) between adsorbent and adsorbate allow the rate of adsorption of alcohols whereas repulsive interactions do not allow the adsorption on the membrane.

\footnotetext{
*For correspondence
}

Accordingly, apart from the pore size, interaction between membrane material and substrate plays an important role in adsorption processes. Understanding the interaction between adsorbent and adsorbate becomes an important factor for designing new adsorbents. Quantum mechanical methods such as Hartree-Fock or density functional theory (DFT) are quite useful in understanding the interactions in molecular systems (hydrogen bonding and electrostatic in the present case). Even though $\beta$-CD drew the attention of many researchers, because of computational cost only a few computational studies have been reported so far. ${ }^{4-16}$ Different methods are used to understand the host-guest interactions in larger systems like complexes of CDs or their derivatives. $^{7-15}$ Recently we have studied CD-alcohol interactions using QM-MM method. ${ }^{16}$ An intermediate value of interaction energy indicates that the rate of adsorption of alcohols on CD membrane is governed by the host-guest interaction. ${ }^{16}$

In recent years, density functional theory (DFT) has proved its applicability to interpret chemical reactivity in complex phenomenon. ${ }^{17}$ Moreover, density functional reactivity theory (DFRT) is used to estimate reactivity parameters. The reactivity descriptors, defined within the framework of DFT are global hardness, global electrophilicity, chemical potential, local softness, Fukui functions, etc. ${ }^{18}$ These descriptors have been tested and studied by several research groups and are found to be very useful in explaining the reactivity patterns in the molecular systems. ${ }^{19-21}$

Recently, a number of theoretical studied have been made to understand the effects of external electric 
field on absorption. ${ }^{22-27}$ Effect of external electric field on hydrogen adsorption over activated carbon separated by dielectric materials was studied by Zhang. ${ }^{23}$ Farmanzadeh and Ghazanfary investigated the interaction between the glycine molecule with the $(6,0)$ zigzag model of single-walled boron nitride nanotubes (BNNTs) with H-terminated at the open end in presence of an external electric field. ${ }^{24}$ Effects of electric field on the adsorption of $\mathrm{CO}$ on gold and graphene nanodot were also studied. ${ }^{25,26}$ Thus, it is expected that the adsorption of the CDs are affected by the presence of external electric field which might affect the filtration process.

Application of external electric field influences both physical and chemical properties of various molecular systems. The effect of electric field on the chemical reactivity has also been carried out in several earlier studies. ${ }^{27-35} \mathrm{Pal}$ and co-workers have studied the behavior of these descriptors in presence of external electric field as well as solvent media. ${ }^{29}$ Chattaraj and co-workers have monitored the effect of electric field on the global and local reactivity indices and confirmed that electric field considerably affects all the local reactivity indices. ${ }^{35}$ Thus, one might expect that the site of attack and selectivity be affected by external electric field.

In the present work, we have considered the CDalcohol adduct and studied the variation of interaction energy in presence of an external electric field. We have attempted to exploit the DFRT to study the variation of their reactivity in presence of such external electric fields. To reduce the computational cost, we have considered only one glucopyranose unit of cyclodextrin molecule.

\section{Theoretical and Computational}

Interaction energy is an important parameter usually used to estimate the stability of a system. However, calculation of the interaction energy in the presence of an external electric field is a challenge to the theoretical chemists. The time-independent non-relativistic electronic Hamiltonian, under the Born-Oppenheimer approximation can be written as:

$$
\hat{H}=-\frac{1}{2} \sum_{i=1}^{N} \nabla_{i}^{2}-\sum_{i, A=1}^{N} \frac{Z_{A}}{r_{i A}}+\sum_{i<j}^{N} \frac{1}{r_{i j}}
$$

In the Hamiltonian the external electric external field is introduced as a perturbation parameter. The dipolar interaction of the Hamiltonian with the electric field, $F$ can be written as,

$$
\hat{H}=\hat{H}_{0}-\hat{d} \cdot \vec{F}
$$

where, $\hat{H}_{0}$ is the unperturbed Hamiltonian and $\hat{d}$ is the dipole moment operator. In the presence of such external perturbation like external electric field the total energy of the system can be writtenas,

$$
E=E_{0}-\sum_{i} d_{i} F_{i}
$$

where, $E_{0}$ is the energy of the system at zero field, $d_{i}$ is the permanent dipole moment and $F_{i}$ is the strength of the field.

Conceptual density functional theory defines the chemical potential $\mu$ as the first derivative of energy with respect to the number of electrons ${ }^{36}$

$$
\mu=\left(\frac{\partial E}{\partial N}\right)_{v(\vec{r})}
$$

and hardness $(\eta)^{37}$

$$
\eta=\frac{1}{2}\left(\frac{\partial^{2} E}{\partial N^{2}}\right)_{v(\vec{r})}=\frac{1}{2}\left(\frac{\partial \mu}{\partial N}\right)_{v(\vec{r})}
$$

where $E$ is the energy and $N$ is the number of electrons of an electronic system at constant external potential, $v(\vec{r})$.

In most numerical applications, chemical potential $(\mu)$ and chemical hardness $(\eta)$ are calculated using finite difference approximation in terms of ionization potential $(I P)$ and electron affinity (EA) and therefore, $\mu$ and $\eta$, given below, can be used as working formulae

$$
\begin{gathered}
\mu=\frac{-(I P+E A)}{2} \\
\eta=\frac{(I P-E A)}{2}
\end{gathered}
$$

Approximations, involving the use of Koopmans' theorem, ${ }^{38}$ defines the $I P$ and $E A$ in terms of the energies of highest occupied molecular orbital $\left(\varepsilon_{\text {HOMO }}\right)$ and lowest unoccupied molecular orbitals $\left(\varepsilon_{L U M O}\right)$

$$
\begin{aligned}
& I P=-\varepsilon_{\text {Номо }} \\
& E A=-\varepsilon_{L U M O}
\end{aligned}
$$

and therefore, $\mu$ and $\eta$ can be expressed as:

$$
\eta=\frac{\varepsilon_{L U M O}-\varepsilon_{H O M O}}{2}
$$

and

$$
\mu=\frac{\varepsilon_{L U M O}+\varepsilon_{H O M O}}{2}
$$

Parr and co-workers proposed electrophilicity $(\omega)$ as a measure of electrophilic power of a ligand and its propensity to soak up electrons. ${ }^{39}$

$$
\left.\omega=\mu^{2} / 2 \eta\right)
$$


The geometrical minima of the species are obtained using 6-31++G(d,p) basis set with Becke three parameter exchange and Lee, Yang and Parr correlation functional (B3LYP) ${ }^{40}$ and is confirmed by the absence of any imaginary frequency. Thereafter single point calculations are carried out at different external electric field values along six directions (along positive and negative directions of the three Cartesian axes, the + sign means that the field is applied along + direction of the axis and - sign means that the field is applied along the - direction of the axis). The range of the strength of external field is chosen from 0.00 a.u. to 0.02 a.u. $\left[1\right.$ a.u. $\left.=51.4 \mathrm{~V} / \AA=51.4 \times 10^{10} \mathrm{Vm}^{-1}\right]$. The global reactivity descriptors (chemical potential, global hardness and electrophilicity) are calculated using equations mentioned above. To check the consistency in results, we repeated our calculation at CAMB3LYP/6-31++G(d,p) level of theory. Choice of the basis set is based on the consideration that in order to obtain reliable properties for hydrogen bonded systems, it is essential to employ basis sets that possess sufficient diffuseness and angular flexibility. All calculations are performed in Gaussian09. ${ }^{41}$

\section{Results and Discussion}

Interaction energy between adsorbent and adsorbate plays the most important role in adsorption process and we emphasized on the effect of variation of electric field on interaction energy between the two. Further, stability of adducts are discussed and validity of the maximum hardness and minimum electrophilicity principle have been tested. ${ }^{42}$

\subsection{Effect of the external electric field on interaction energy}

To examine the effect of external electric field on interaction energy between $\mathrm{CD}$ and alcohols, initially we have calculated the interaction energy in absence of any external electric field. Super molecular approach has been adopted in calculating interaction energy [for $\mathrm{A}+$ $\mathrm{B} \rightarrow \mathrm{AB}, \Delta E_{\text {int }}=\left(E_{\mathrm{AB}}\right)-\left(E_{\mathrm{A}}+E_{\mathrm{B}}\right)$, where, $E$ is the total energy of the corresponding species]. In all cases, we have taken care of basis set superposition error (BSSE) using counterpoise correction method ${ }^{43,44}$ Calculated BSSE corrected gas phase interaction energies in adducts (figure 1) are found to be $-33.737,-33.597$ and $-33.552 \mathrm{kcal} / \mathrm{mol}$ for CD-methanol, CD-ethanol and CD-butanol respectively at B3LYP/631++G(d,p) level of theory. Effect of external electric field on the interaction energy is presented in figures 2 and 3.

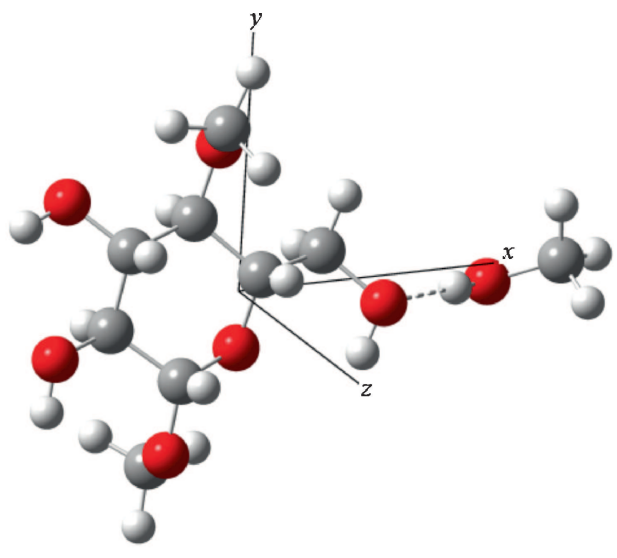

(a) CD-Methanol

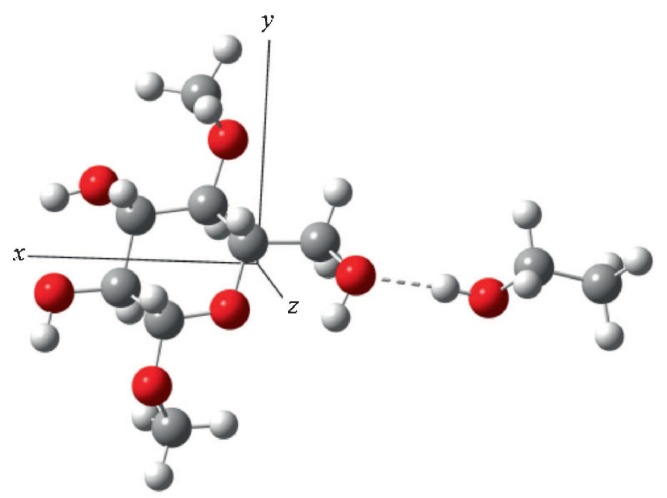

(b) CD-Ethanol

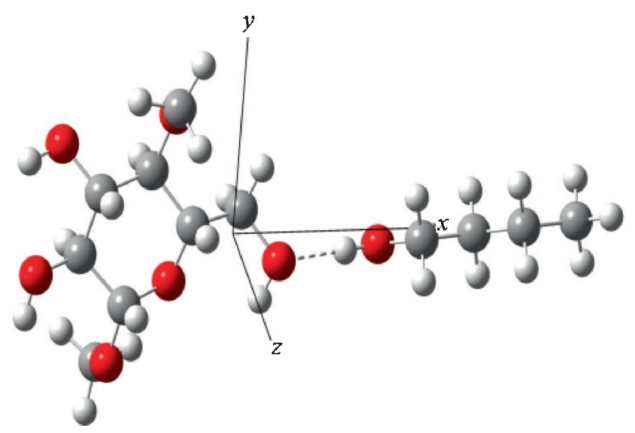

(c) CD-Butanol

Figure 1. Optimized structure of $\mathrm{CD}$-alcohol adduct at B3LYP/6-31++G(d,p) level of theory.

On applying an electric field upon the CD-alcohol adducts, interaction energy is observed to influence significantly and the variation is not same along all the directions. On application of the electric field along $-x$ direction, interaction energy in case of ethanol-CD adduct is observed to increase (more negative), imparting stability to the adduct. Interaction energy is calculated to be $-39.11 \mathrm{kcal} / \mathrm{mol}$ in presence of field of strength of 0.01 a.u. applied along $-x$ direction, figure 2a. Variation of interaction energy in case of CD-methanol and CD-butanol adducts are opposite to that of CD- ethanol adduct; exhibited more interactions 


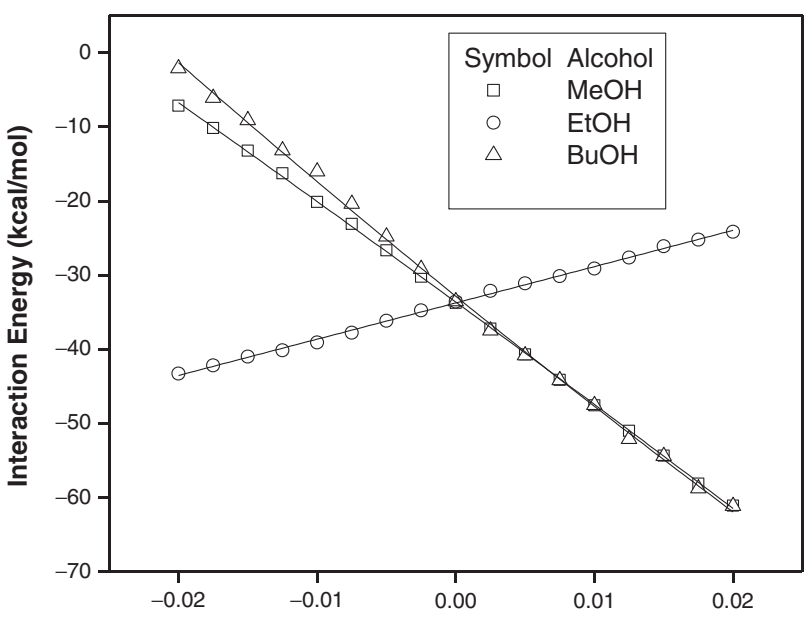

(a)

Electric field (a.u.)

along $x$-axis

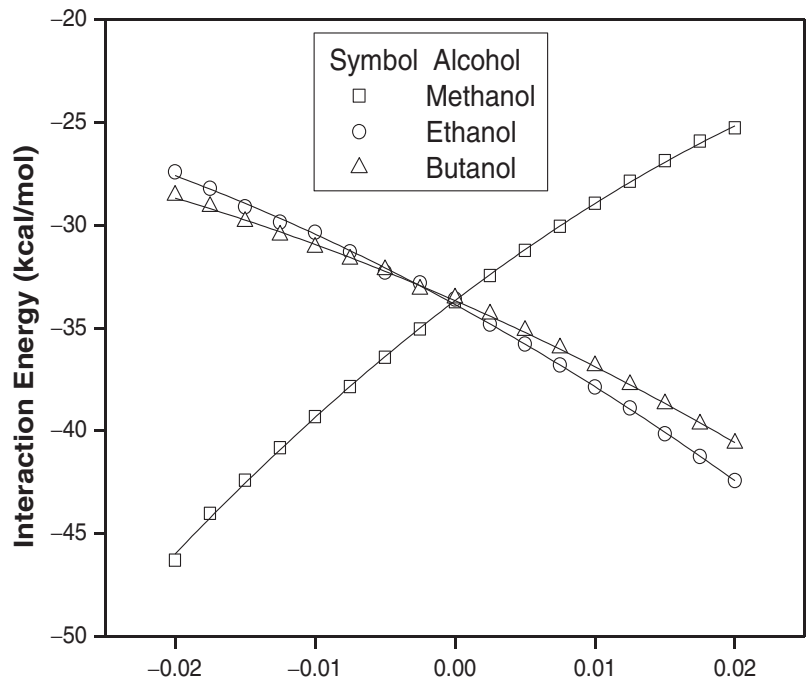

(b)

Electric field (a.u.)

along $y$-axis

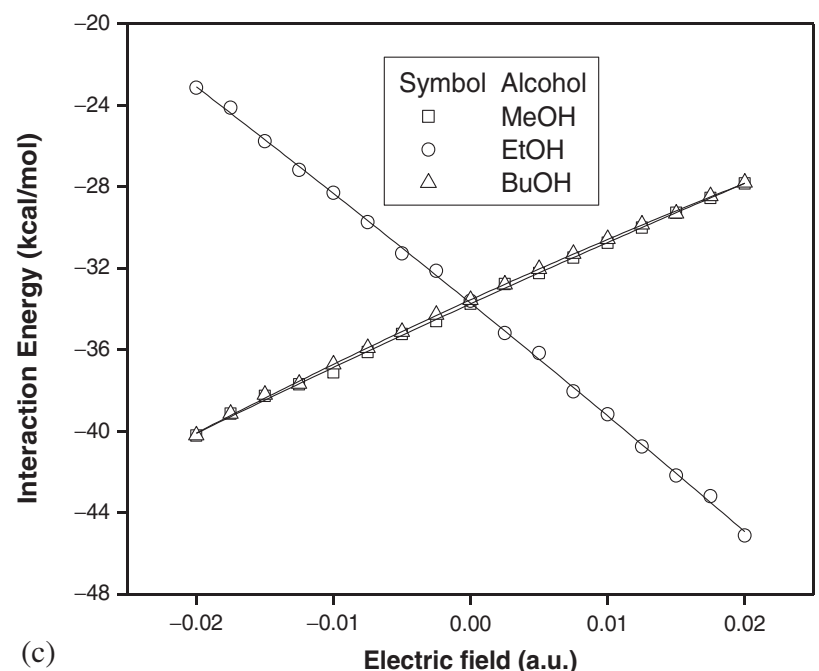

along $z$-axis

Figure 2. Variation of interaction energy along (a) $x$-axis, (b) $y$-axis and (c) $z$-axis with external electric field at B3LYP/6-31++G(d,p) level of theory.

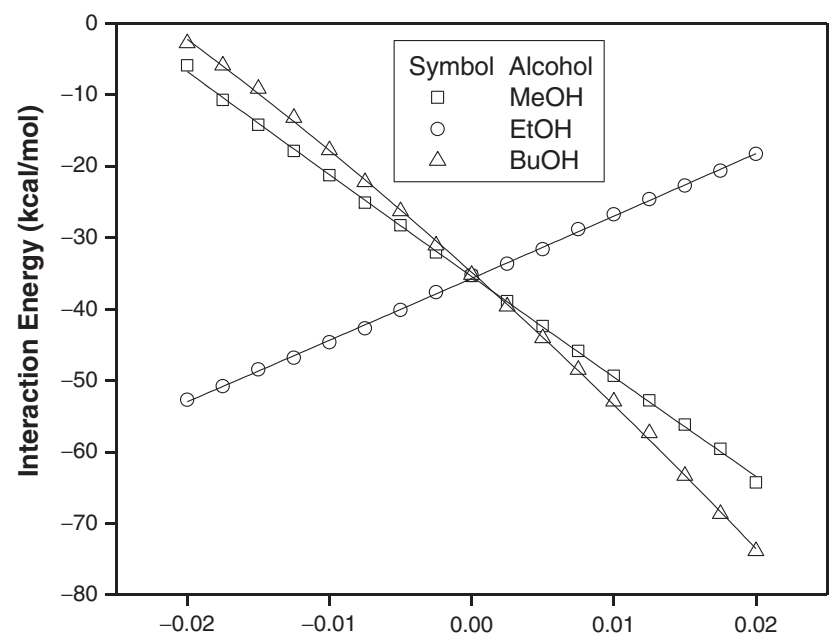

(a)

Electric field (a.u.)

along $x$-axis
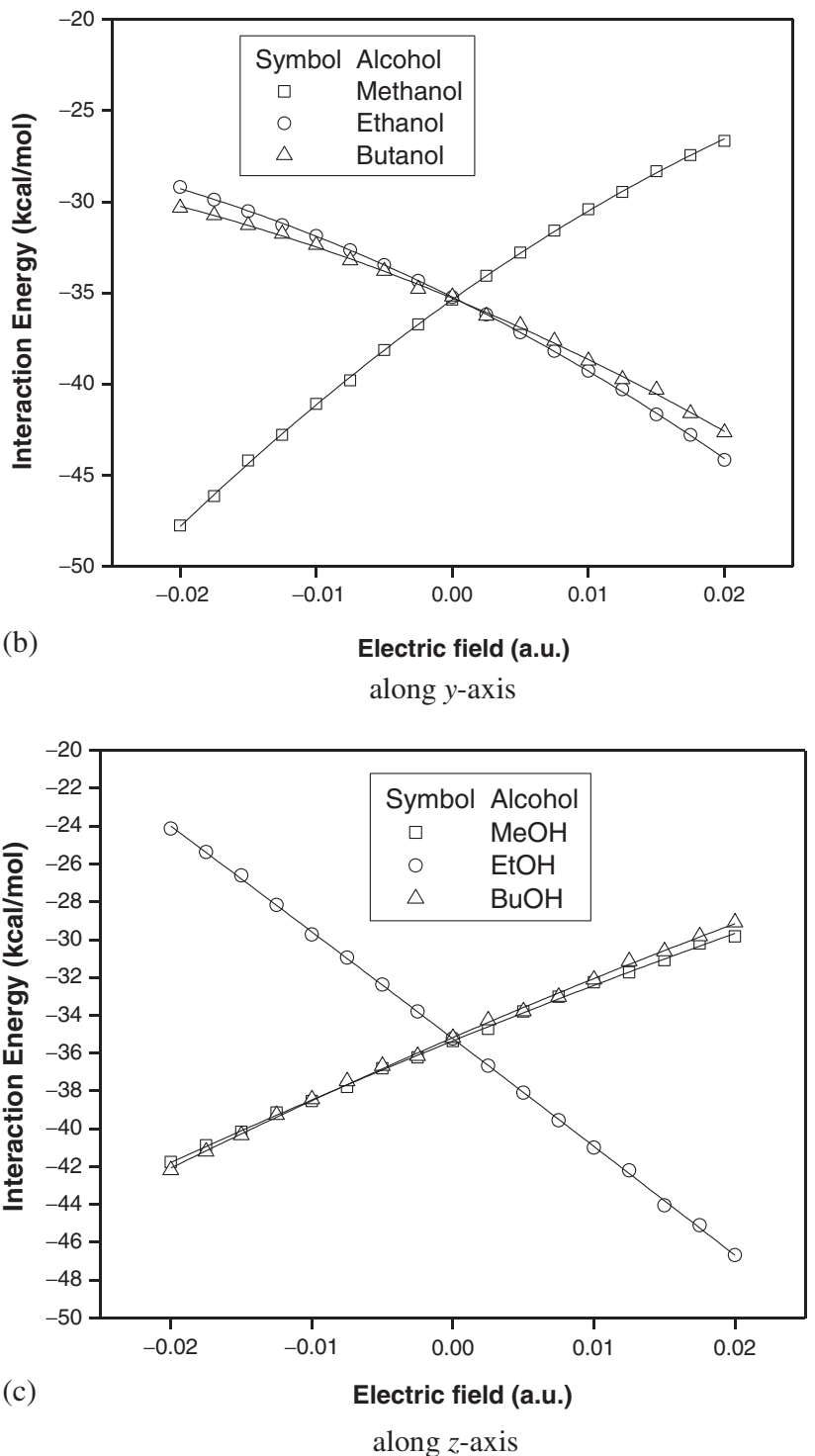

Figure 3. Variation of interaction energy along (a) $x$-axis, (b) $y$-axis and (c) $z$-axis with external electric field at CAMB3LYP/6-31++G(d,p) level of theory. 
on application of the field along $+x$ direction. Interaction energy is $-47.583 \mathrm{kcal} / \mathrm{mol}$ and $-47.530 \mathrm{kcal} / \mathrm{mol}$ in presence of field of strength of 0.01 a.u. for CDmethanol and CD-butanol respectively and opposite trend resulted along $-x$ direction (figure 2a). Thus, the results confirmed the sensitivity of the interaction energy toward the direction of the applied field. In addition, application of the field along $-z$ direction stabilizes CD-methanol and CD-butanol adducts and reverse is the case for $\mathrm{CD}$-ethanol adduct. Exactly same trend is observed in both the cases (CD-methanol and CD-butanol adducts) figure 2c. On the other hand, along $y$-axis, variation of interaction energy in case of CD-methanol adduct is opposite to that of CD-ethanol and CD-butanol adducts (figure 2b). Same results were observed in CAM-B3LYP/6-31++G(d,p) level of theory (figure 3).

\subsection{Variation of the global reactivity descriptors}

Stability of adducts in presence an external electric field is studied using their global hardness values by varying the field strength, results are presented in figure 4. The global hardness values are found to be 0.117 a.u., 0.116 a.u. and 0.115 a.u. for CD-methanol, CD-ethanol and CD-butanol respectively in absence of electric field. When the external electric field is varied from -0.02 a.u. to +0.02 a.u. along the two axes, the global hardness passes through a maximum and the maxima occurs at around zero field value. Thus according to maximum hardness principle, (maximum hardness refers to maximum stability according to maximum hardness principle) the $\mathrm{CD}$-alcohol adduct attains maximum stability when the external field is absent. Hardness is found to be 0.053 a.u., 0.052 a.u. and 0.029 a.u. for
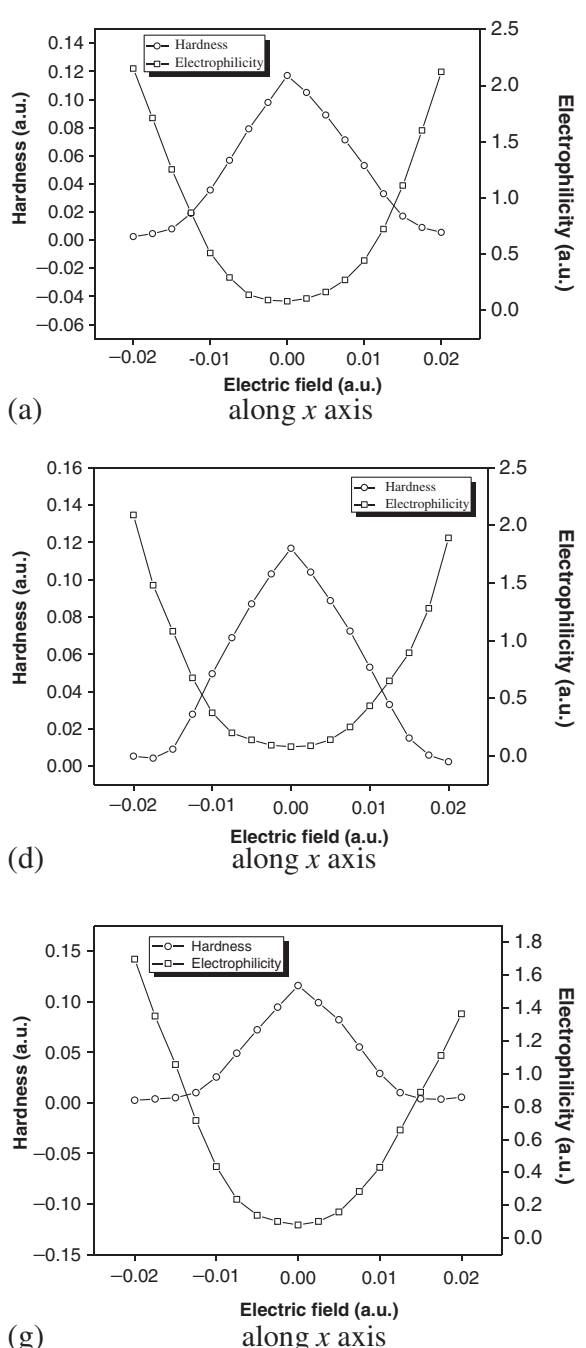

(g)

Figure 4. Variation of global hardness, electrophilicity with external field at B3LYP/6-31++G(d,p) level of theory (a) $-(\mathbf{c})$ CD-Methanol along $x, y$ and $z$ axis, (d)-(f) CD-ethanol along $x, y$ and $z$ axis, (g)-(i) CD-butanol along $x, y$ and $z$-axis.
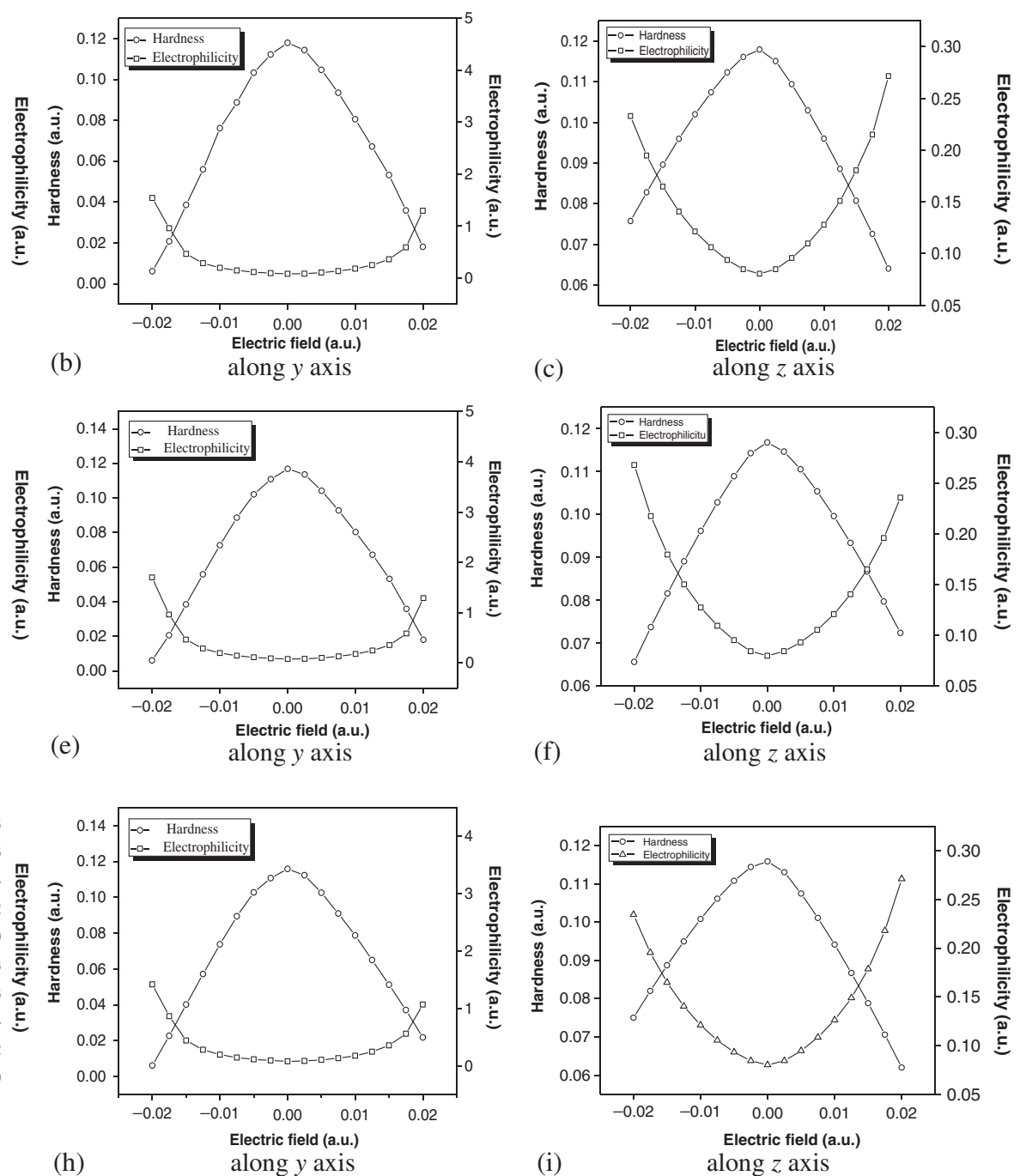
CD-methanol, CD-ethanol, and CD-butanol respectively in presence of field strength of 0.01 a.u. along $+x$ direction. The corresponding values along $+y$ direction and $+z$ direction are 0.0804 a.u., 0.0802 a.u., 0.078 a.u. and 0.095 a.u., 0.099 a.u., 0.094 a.u. respectively. The variation of hardness along both directions of $y$ axis and $z$-axis is found to be similar to that along $x$ axis. On the other hand, as the value of external electric field increases, irrespective of the direction of the field, electrophilicity of the adduct increases in both axes, figure $4 \mathrm{a}-\mathrm{f}$. The electrophilicity values found to be 0.0803 a.u., 0.079 a.u. and 0.0801 a.u. for CDmethanol, CD-ethanol and CD-butanol respectively. In presence of field of strength of 0.01 a.u. along $+x$ direction, electrophilicity values obtained are 0.443 a.u., 0.433 a.u. and 0.430 a.u. for CD-methanol, CD-ethanol and CD-butanol respectively. Along $+y$ and $+z$ direction, the electrophilicity values found to be 0.1808 a.u.,
0.1803 a.u. 0.176 a.u. and 0.127 a.u., 0.120 a.u., 0.26 a.u. for the corresponding adducts which are comparatively lesser than along $+x$ direction. It is observed that the electrophilicity passes through a minimum when the external electric field is varied and the minima occurs at zero field value; satisfying minimum electrophilicity principle. Results clearly indicate that the CD-alcohol adducts become unstable in presence of external electric field which might affect the filtration process. Similar results are observed in CAM-B3LYP/6-31++G(d,p) level of theory (figure 5).

\subsection{Variation of HOMO energy $\left(E_{\text {Номо }}\right)$ with applied field strengths}

Variation of $\mathrm{E}_{\mathrm{HOMO}}$ of the $\mathrm{CD}$-alcohol with the applied field strengths is presented in figure $6 . \mathrm{E}_{\text {Hомо }}$ is found to be 0.255 a.u., 0.253 a.u. and 0.252 a.u. for

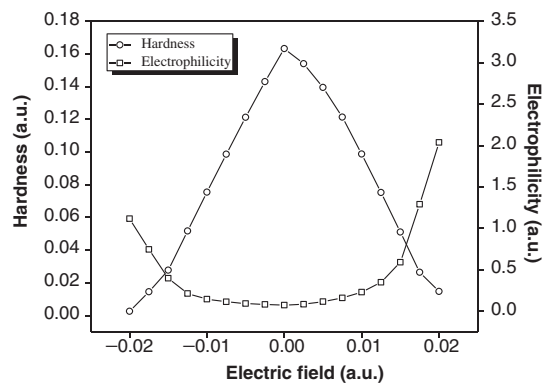

(a)
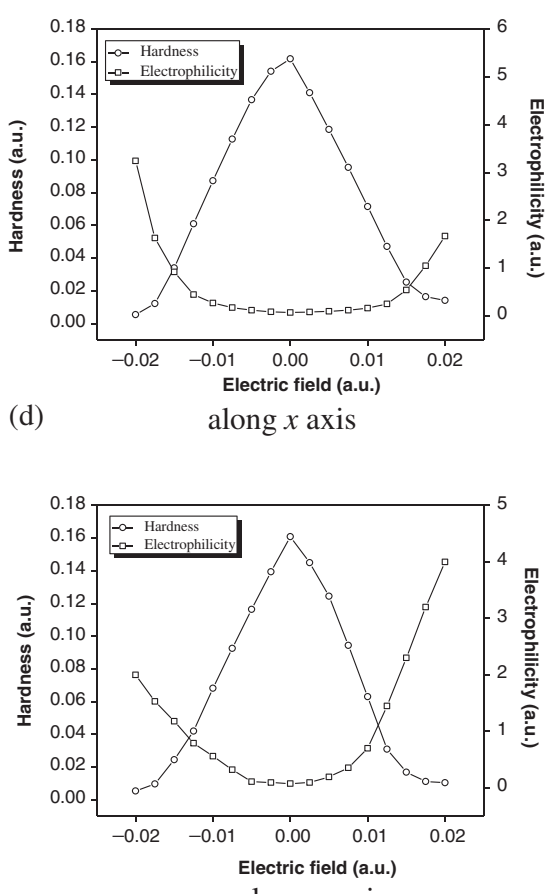

(g)
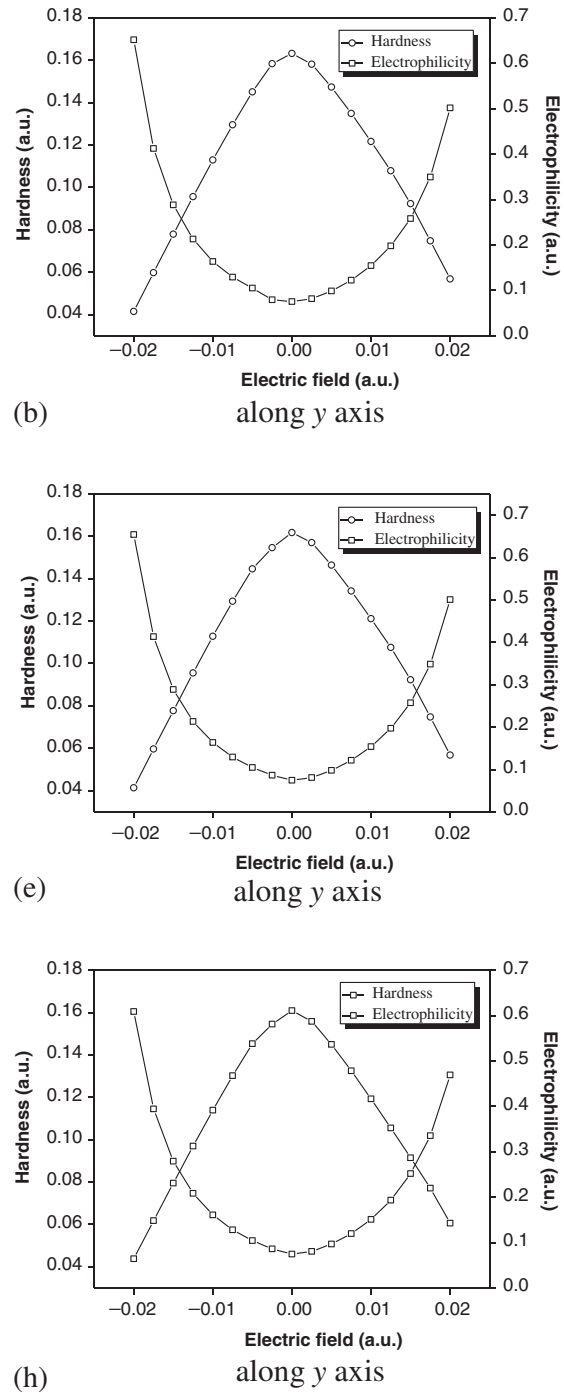

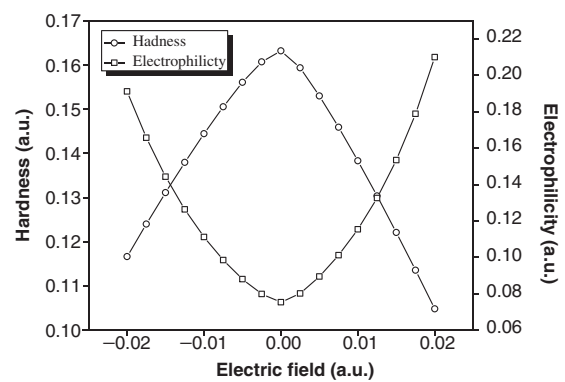

(c)

along $z$ axis

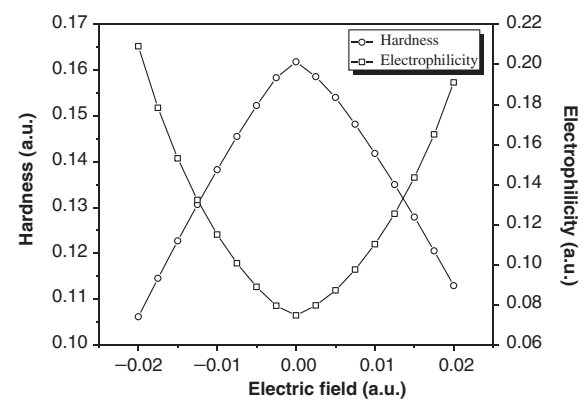

along $z$ axis

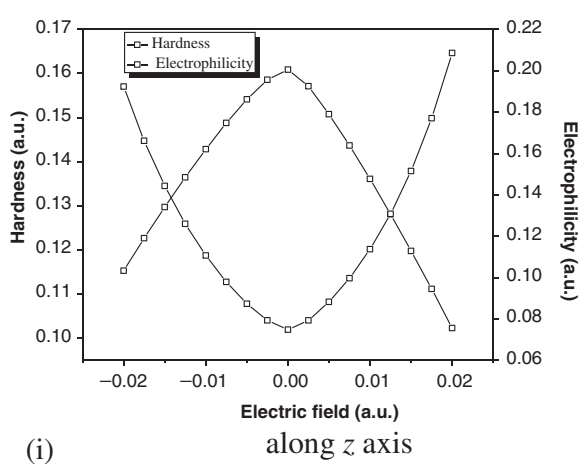

(i)

Figure 5. Variation of global hardness, electrophilicity with external field at CAMB3LYP/6-31++G(d,p) level of theory (a)-(c) CD-Methanol along $x, y$ and $z$ axis, (d)-(f) CD-ethanol along $x, y$ and $\mathrm{i}$ axis, (g)-(i) CD-butanol along $x, y$ and $z$-axis. 


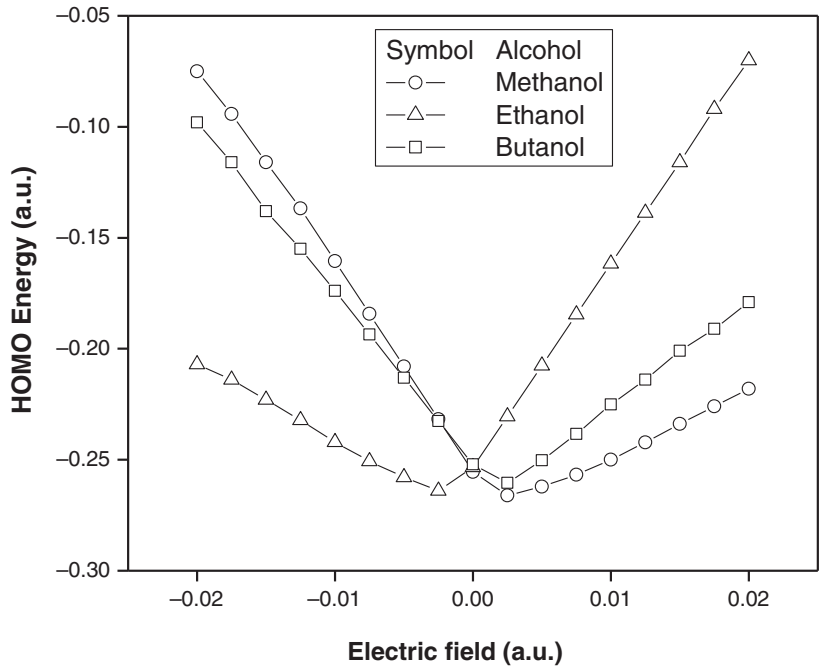

(a)

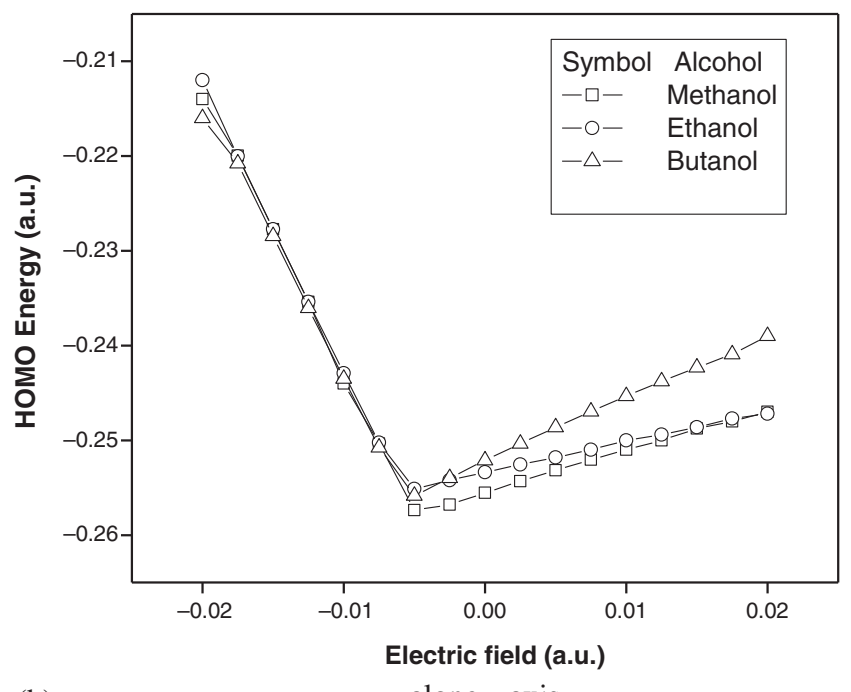

(b)

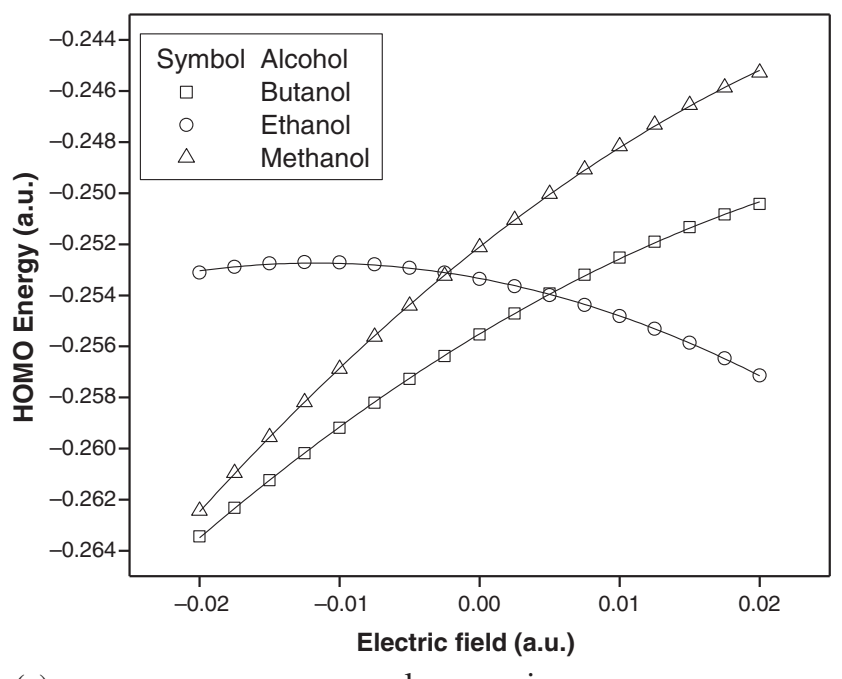

(c)

along $z$ axis

Figure 6. Variation of HOMO Energy (a.u.) of CD-alcohols along (a) $x$-axis, (b) $y$-axis and (c) $z$-axis with external electric field at B3LYP/6-31++G(d,p) level of theory.

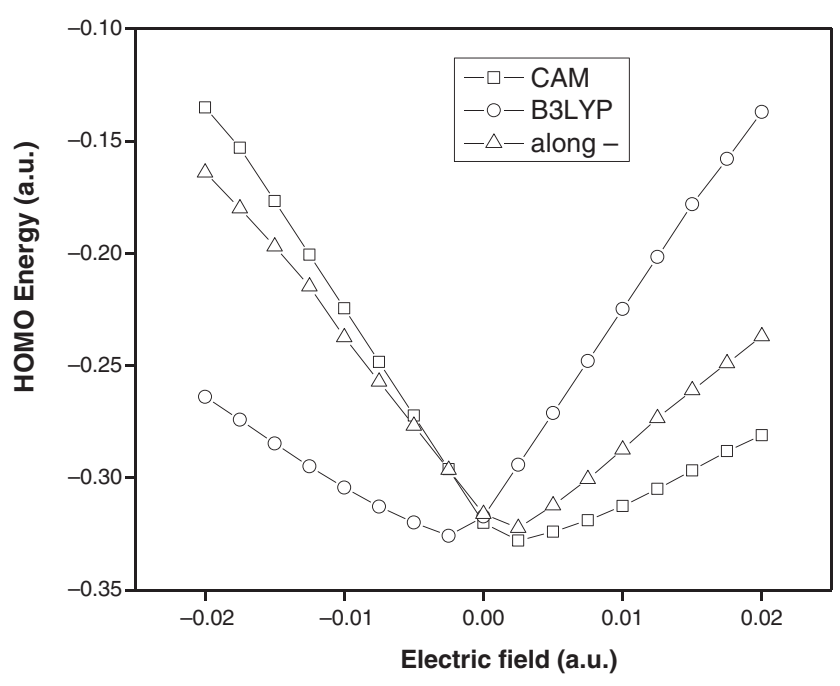

(a)
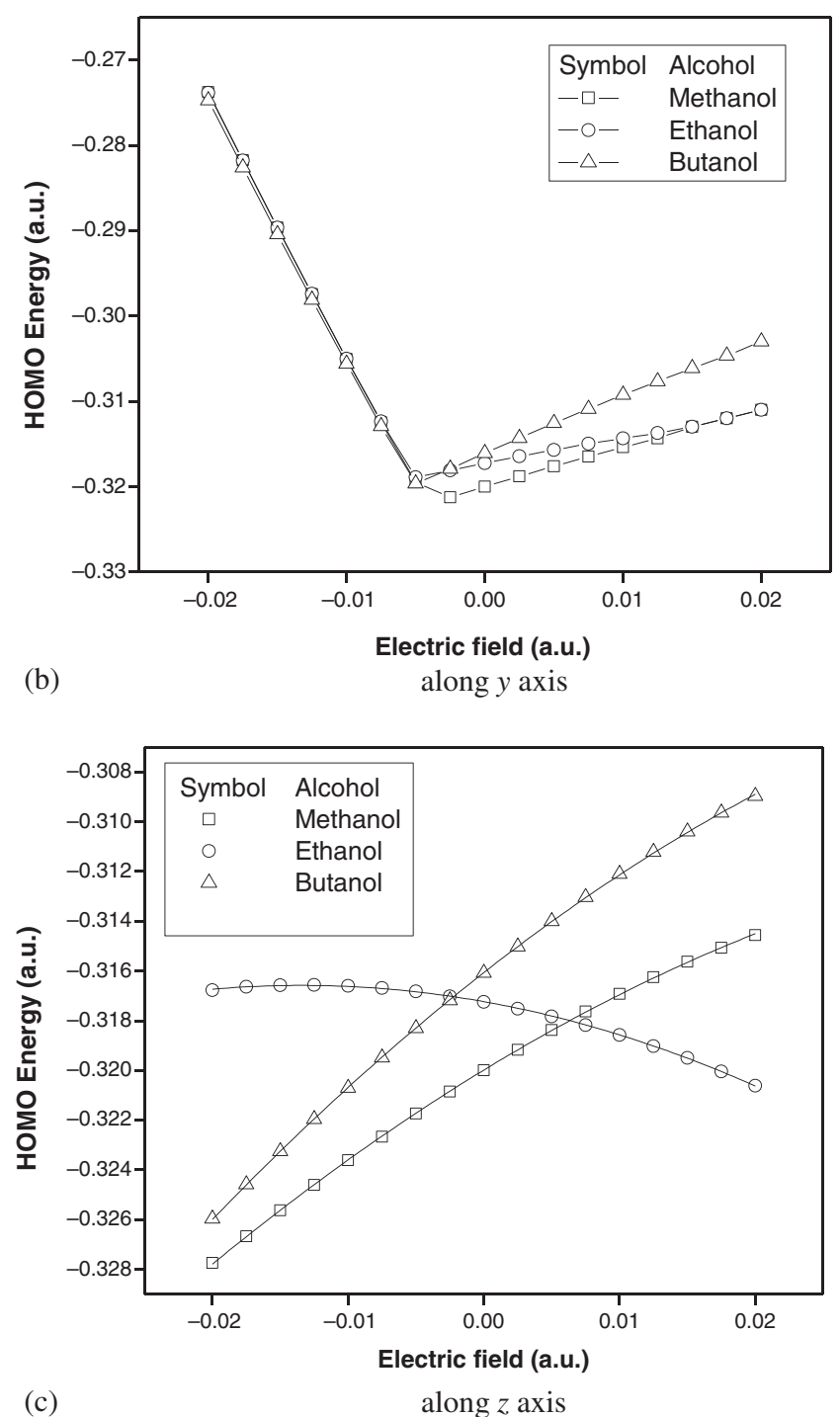

Figure 7. Variation of HOMO Energy (a.u.) of CD-alcohols along (a) $x$-axis, (b) $y$-axis and (c) $z$-axis with external electric field at CAMB3LYP/6-31++G(d,p) level of theory. 
CD-methanol, CD-ethanol and CD-butanol respectively in absence of electric field. Figure 6 advocates a significant impact of the strength of the applied electric field.

However, the variation is not same along all the directions. Upon application of the electric field along $-x$ direction, $\mathrm{E}_{\text {НОмо }}$ in case of $\mathrm{CD}$-ethanol adduct is observed to decrease (more negative), imparting overall stability to adducts. HOMO energy is -0.241 a.u. in presence of field of strength 0.01 a.u. along $-x$ direction, figure $6 \mathrm{a}$. Variation of HOMO energy in case of CD- methanol and CD- butanol adducts is opposite to that of CD- ethanol adduct $(-0.160$ a.u. and -0.173 a.u. for CD- methanol and CD- butanol respectively) figure $6 \mathrm{a}-\mathrm{c}$. The results confirmed the sensitivity of the HOMO energy toward the direction of the applied field. Observations along $y$-axis show the same trend as along $x$-axis. In addition, application of the field along $-z$ direction stabilizes CD- methanol and CD- butanol adducts and reverse is the case for CD- ethanol adduct. Similar observations were made in CAM-B3LYP/6-31++G(d,p) level of theory (figure 7).

\section{Conclusions}

We studied the response of CD-alcohol adduct towards external electric fields. Our study was mainly focused on the variation of interaction energy with the variation of electric field and is observed to depend on the strength and direction of the applied field. Additionally, we analyzed the variation in the stability and reactivity of the CD-alcohol adducts using density based reactivity descriptors. Moreover, stability and $E_{\text {НОмо }}$ were observed to be responsive towards the strength and direction of the applied electric field. Our observations reveal that the stability of CD-alcohol adduct is highly sensitive towards electric field. The observations advocate that one can tune the filtration of alcohols through CD composite membrane.

\section{Supplementary Information}

Cartesian coordinates of the CD-alcohol complexes are given in table table S1-S3. Normal modes of the complexes are provided in table S4. Supplementary Information is available at www.ias.ac.in/chemsci.

\section{References}

1. Loftsson T and Duchene D 2007 Int. J. Pharm. 3291

2. Baruah K, Hazarika S, Borthakur S and Dutta N N 2012 J. Appl. Polym. Sci. 1253388

3. Baruah K and Hazarika S 2014 J. Appl. Polym. Sci. 131 40537
4. Stachowicz A, Styrcz A, Korchowiec J, Modaressi A and Rogalski M 2011 Theor. Chem. Acc. 130939

5. Britto M A F O, Nascimnento C S Jr. and Dos Santos H F 2004 Quim. Nova 27882

6. Pinjari R V, Joshi K A and Gejji S P 2007 J. Phys. Chem. A 11113583

7. Stachowicz A, Rogalski M and Korchowiec J $2013 \mathrm{~J}$. Mol. Model 194163

8. Tokes B, Ferencz L, Buchwald P, Donath-Nagy G, Vancea S, Santa N and Kis E L 2008 J. Biochem. Biophys. Methods $\mathbf{7 0} 1276$

9. Tablet C and Hillebrand M 2008 Spectrochim. Acta Part A 70740

10. Zhou Z M, Li X, Chen X P, Fang M and Dong X 2010 Talanta 82775

11. Raffaini G, Ganazzoli F, Malpezzi L, Fuganti C, Fronza G, Panzeri W and Hele A 2009 J. Phys. Chem. B 113 9110

12. Gotsev M G and Ivanov P M 2009 J. Phys. Chem. B 113 5752

13. Lipkowitz K B and Stoehr C M 1996 Chirality 8341

14. Zuchowski G, Zborowski K and Bojarski J 2000 Chem. Anal. 45767

15. Zborowski K and Zuchowski G 2002 Chirality 14632

16. Baruah K, Sinha S, Hazarika S and Bhattacharyya P K 2015 J. Macromol. Sci. Pure Appl. Chem. 5264

17. (a) Kohn W, Becke A D and Parr R G 1996 J. Phys. Chem. 100 12974; (b) Baerends E J and Gritsenko O V 1997 J. Phys. Chem. A 101 5383; (c) Chermette H 1998 Coord. Chem. Rev. 178 699; (d) Andrews L and Citra A 2002 Chem. Rev. 102 885; (e) Ziegler T and Autschbach J 2005 Chem. Rev. 105 2695; (f) Neese F 2009 Coord. Chem. Rev. 253 526; (g) Schultz N E, Zhao Y and Truhlar D G 2005 J. Phys. Chem. A 10911127

18. (a) Parr R G and Yang W 1989 In Density Functional Theory of Atoms and Molecules (New York: Oxford University Press); (b) Melin J, Aparicio F, Subramanian V, Galvan M and Chattaraj P K 2004 J. Phys. Chem. A 108 2487; (c) Cohen M H and Wasserman A 2007 J. Phys. Chem. A 111 2229; (d) Ramya K R and Venkatnathan A 2012 J. Phys. Chem. A 1167742; (e) Pearson R G 1993 Acc. Chem. Res. 26 250; (f) Parr R G and Zhou Z 1993 Acc. Chem. Res. 26 256; (g) Pearson R G 1997 In Chemical Hardness (Weinheim: John WileyVCH); (h) P K Chattaraj (Ed.) 2009 In Chemical Reactivity theory (Boca Raton: CRC press); (i) Parr R G and Pearson R G 1983 J. Am. Chem. Soc. 105 7512; (j) Parr R G, Von Szentpaly L and Liu S B 1999 J. Am. Chem. Soc.121 1922; (k) Pearson R G 1987 J. Chem. Educ.64 561; (1) Parr R G and Chattaraj P K 1991 J. Am. Chem. Soc. 113 1854; (m) Morell C, Grand A and Toro-Labbe A 2005 J. Phys. Chem. A 109 205; (n) Morell C, Grand A and Toro-Labbe A 2006 Chem. Phys. Lett. 425 342; (o) Berkowitz M 1987 J. Am. Chem. Soc. 109 4823; (p) De Proft, F, Ayers P W, Fias S and Geerlings P J 2006 Chem. Phys. 125 214101; (q) Ayers P W, Morell C, De Proft F and Geerlings P 2007 Chem. Eur. J. 138240

19. (a) Chandra A K and Nguyen M T 2007 Faraday Discus. 135 191; (b) Molteni G and Ponti A 2003 Chem. Eur. J. 9 2770; (c) Roy R K 2003 J. Phys. Chem. A 107 397; (d) Nguyen H M T, Peeters J, Nguyen M T and Chandra A K 2004 J. Phys. Chem. A 108 484; (e) TorrentSucarrat M, De Proft F, Ayers P W and Geerlings P 2010 
Phys. Chem. Phys. Chem. 12 1072; (f) Chattaraj P K and Duley S 2010 J. Chem. Eng. Data 551882

20. (a) Geerlings P, De Proft F and Langenaekar W 2003 Chem. Rev. 103 1793; (b) Chattaraj P K, Sarkar U and Roy D R 2006 Chem. Rev. 106 2065; (c) Parthasarathi R, Subramanian V, Roy D R and Chattaraj P K 2004 Bioorg. Med. Chem. 12 5533; (d) Parthasarathi R, Padmanabhan J, Elango M, Subramanian V and Chattaraj P K 2004 Chem. Phys. Lett. 394 225; (e) Roy R K 2004 J. Phys. Chem. A 108 4934; (f) Torrent-Sucarrat M, Luis J M, Duran M and Sola M 2002 J. Chem. Phys. 11710561

21. (a) Roy R K and Saha S 2010 Annu. Rep. Prog. Chem. Sect. C: Phys. Chem. 106 118; (b) Vijayaraj R, Subramanian V and Chattaraj P K 2009 J. Chem. Theor. Comp. 5 2744; (c) Putz Mihai V and Mingos D Michael P. (eds.) 2012 In Applications of Density Functional Theory to Chemical Reactivity (Berlin: Springer)

22. Rastegar S F, Hadipour N L, Tabar M B and Soleymanabadi H 2013 J. Mol. Model. 193733

23. Zhang Z 2012 In Effect of External Electric Field on Hydrogen Adsorption Over Activated Carbon Separated by Dielectric Materials, Ph.D Thesis (US: Michigan Technological University)

24. Farmanzadeh D and Ghazanfary S 2013 J. Serb. Chem. Soc. 7875

25. Koverga A A, Frank S and Koper M T M 2013 Electrochimica Acta 101244

26. Liu H and Lee J Y 2012 Phys. Chem. C 1163034

27. Brooks P R and Jones M E 1966 J. Chem. Phys. 453449

28. (a) Abronin A, Vorontsova I K and Mikheikin I D 2003 Chem. Phys. Lett. 368 523; (b) Gevirzman R and Kozirovski J 1972J. Chem. Soc. Faraday Trans. 2(68) 1699

29. (a) Kar R, Chandrakumar K R S and Pal S 2007 J. Phys. Chem. A 111 375; (b) Kar R and Pal S 2008 Theor.
Chem. Acc. 120 375; (c) Kar R and Pal S 2010 Int. J. Quant. Chem. 110 1642; (d) Kar R and Pal S 2008 In External Field and Chemical Reactivity P K Chattaraj (ed.) (Boca Raton: CRC Press)

30. Saban K V, Thomas J, Varughese P A and Varghese G 2002 Cryst. Res. Technol. 371188

31. Jaroszeski M J, Heller R and Gilbert R2000 In Methods in Molecular Medicine (Totowa, NJ: Humana Press)

32. Bandrauk A D, Sedik E S and Matta C F 2004 J. Chem. Phys. 1217764

33. Hirao H, Chen H, Carvajal M A, Wang Y and Shaik S 2008 J. Am. Chem. Soc. 1303319

34. Tarquini A 2009 In The Effect of External Electric Fields on Biological Systems: Theoretical Computational study Ph.D thesis (Universitá Degli Studi di Roma: Sapienza)

35. Parthasarathi R, Subramanian V and Chattaraj P K 2003 Chem. Phys. Lett. 38248

36. Parr R G, Donnelly R A, Levy M and Palke W E 1978 J. Chem. Phys. 683801

37. Parr R G and Pearson R G 1983 J. Am. Chem. Soc. 105 7512

38. Koopmans T 1934 Physica 1104

39. Parr R G, Szentpaly L V and Liu S 1999 J. Am. Chem. Soc. 1211922

40. (a) Becke A D 1993 J. Chem. Phys. 98 5648; (b) Lee C, Yang W and Parr R G 1988 Phys. Rev. B 37 785

41. Frisch M J et al. 2010 Gaussian 09, Revision B.01, Gaussian, Inc., Wallingford, CT

42. Parr R G and Chattaraj P K 1991 J. Am. Chem. Soc. 113 1854

43. Boys S F and Bernardi F 1970 Mol. Phys. 19553

44. Simon S, Duran M and Dannenberg J J 1996 J. Chem. Phys. 10511024 